and the perception of pleasurable sensations by the brain. Yet we have little understanding of how the two components relate to each other. Moreover, a distinction is often made between clitoral and vaginal orgasm. Whether these are physiologically different - let alone whether they evolved under different selection pressures - is unknown. Indeed, the neuroanatomy of the genital region is poorly understood, and we have scant data on how much it varies among women. Brain scans suggest that different parts of the brain may be involved in orgasm for males and females - which would be consistent with natural selection acting on females directly - but the sample sizes are as yet too small to draw confident conclusions.

To understand the significance of the varia- tion in women's experience of orgasm, we need to know what causes this variation. Is it due to genetic differences in genital anatomy? To differences in the way brains perceive pleasure? To psychological or cultural factors? Or to a physical incompatibility between a woman and her partner(s)? In other words, do some women lack a capacity for orgasm, or is the capacity there but never realized? Again, data are lacking. A recent twin study (K. M. Dunn et al. Biol. Lett. doi:10.1098/rsbl.2005.0308; 2005) suggests there may be a genetic component - if your identical twin has orgasms it's likely that you do too - but whether the effect is due to physiology or psychology is unclear.

And we need to know more about when other primates experience orgasm. Do females in other species have orgasms with some males but not with others? No one knows. Here, too, we need to know how pelvic contractions translate into brain waves. And we need to investigate males as well as females: it is often simply assumed that males in other species have orgasms. Data on other primates will help us to understand the relationship between male and female orgasm, and whether the female orgasm evolved before the split between humans, chimpanzees and bonobos.

In short, it's time to collect data. Without it, the debate will remain like sex sometimes is: furious, empty and anticlimactic.

Olivia P. Judson is a research fellow in evolutionary biology at Imperial College, Prince Consort Road, London SW7 2BW, UK.

\section{Prussian precision}

\section{Anton Hallmann's technical drawings brought geometry to life.}

\begin{abstract}
Martin Kemp
Technical drawing lay at the heart of a flourishing of both the arts and the physical sciences in nineteenth-century Berlin. The precise lines of geometry obtained practical expression and gave shape to painting, sculpture and architecture. Yet the measured precision it allowed provided the basis for engineering and instrument-making, even the science of warfare. The mastery of perspective projection and the geometrical casting of shadows was central to it all. The drawings of the architect Anton Hallmann - on show in the exhibition Apoll im Labor at the Berlin Museum of Medical History until 2 October - exemplify the heights reached by technical draftsmanship in the nineteenth century. Introduced to projective geometry at the Artillerieschule in Hanover, Hallmann became a master of precise architectural representation, and was best known for his depiction of buildings of classical antiquity. His drawings range from the meticulous perspectival rendering of the whole and parts of buildings to extraordinary abstract exercises in which he set geometrical bodies in measured spaces under specific illumination.
\end{abstract}

In the example shown here, he scatters a series of bodies across a stage-like ground on which two torches provide what are taken to be point sources of light. The shadows are observed in full geometric projection, as they cast a complex ensemble of angular and conic contours across the space. The relative intensities of the two overlapping shadow systems are meticulously computed, with often surprising results.

Hallmann's link with science is his friendship with Emil Du Bois-Reymond, the pioneer of electrophysiology and inventor of instruments for measuring biological forces. There is an obvious connection through Hallmann's drawings of the mechanical system of bones and muscles in the human body, but the affinity goes deeper than a common subject. What they and other Berlin practitioners of the arts and sciences shared was a passion for classical clarity.

Du Bois-Reymond depicted himself in an engraving as a semi-nude classical god using his Multiplikator - a precision galvanometer - to measure his muscular electricity (see Nature 436, 27; 2005). He designed his own instruments, which were constructed and operated with a love of form and space in which the beauty of pure mathematics was conjoined with the practical measurement of the forces of nature. This was euclidian mathematics embodied in human form, as exemplified in ancient sculpture.

The network of relationships extends to
Du Bois-Reymond's fellow members of the Berlin Physical Society, the psychologist Ernst Brücke and the physicist Hermann von Helmholtz, both of whom were keenly involved in the visual arts. Du Bois-Reymond had harboured ambitions to be a painter and taught anatomy to the students in Berlin's art academy. They all shared a vivid sense of the aesthetics of the powers of nature, as revealed through measurement and charted most potently on the curves of their graphs. In Hallmann's primary field of architecture, the net extends to the classicist Karl Friedrich Schinkel, master builder and engineer, and his students at the Bauakademie, whose building he designed in a semi-industrial style in 1831. In the perspectival depiction of cityscapes, the net embraces Eduard Gärtner, master-painter of Berlin panoramas, and Johann Hummel, famed for his paintings' optical exactitude (Nature 395, 649; 1998).

Within this Berlin nexus, whether you start with a physicist or a painter, you can connect to any other discipline in just two or three moves. No division into 'two cultures' here. Martin Kemp is professor of the history of art at the University of Oxford, Oxford OX11PT, UK.

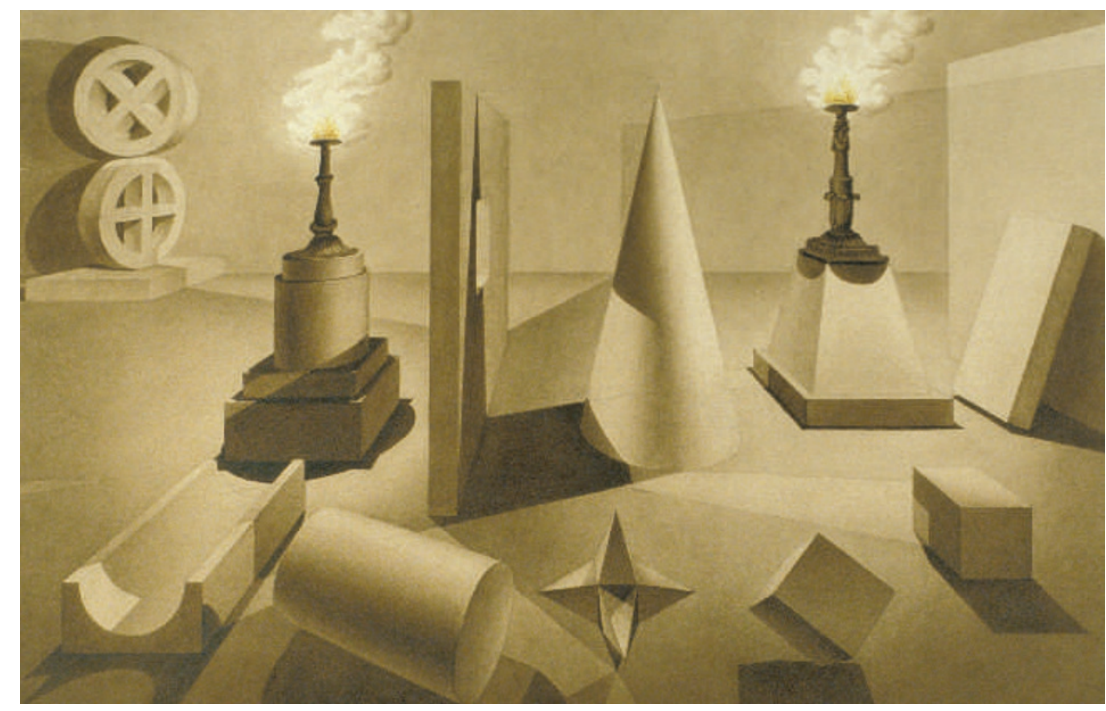

